\title{
A Comprehensive Study of the Functions and Operations of Control Systems
}

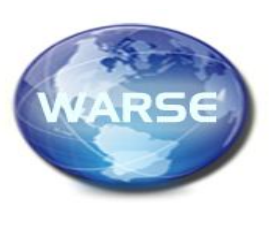

Aaron Don M. Africa, Patrick Bernard T. Arevalo, Arsenic S. Publico, Mharela Angela A. Tan

Department of Electronics and Communications Engineering

De La Salle University, Manila

2401 Taft Ave., Malate, Manila 1004, Philippines

aaron.africa@dlsu.edu.ph

\begin{abstract}
A control system is used to achieve the required feedback by controlling the output. Control systems are widely used in everyday devices such as traffic lights and cars or even processes. These systems help in further understanding and improving the process that is being utilized. It also represents the process in a much more straightforward manner that makes it easier to comprehend. This paper aims to broaden the insight on control systems and to discuss different control systems that are being used.
\end{abstract}

Key words: Control Systems, System Block Diagrams, Feedback Systems, Block Diagrams.

\section{INTRODUCTION}

Control is what we do to change or improve the operation of any kind of system in order to achieve a particular result or desired process. Examples of common quantities modified in systems are speed, temperature, voltage, and amplitude [1]

When various elements and components are combined to create a system producing the desired output, the said system is considered to be a control system. Control systems are composed of devices or a set of devices which manipulates the behavior of other devices or systems to obtain the desired output. These systems require a transparent and logical-mathematical relationship between the input and output of the system.

Because of the rise of modern technology, it can be said that almost every aspect of our daily routines is caused or affected by control systems. Automobiles, refrigerators, air conditioners, including many more home appliances are examples of control systems. Since control systems play a major role in human lives and activities, designs of these systems must observe criteria such as accuracy, sensitivity, stability, noise, bandwidth, speed, and oscillation. [2]

There are two primary types of control systems: the open loop control system and the closed-loop control system. In an open loop control system, the control action is not dependent on the output of the system. Some of the known advantages of open-loop control systems are simplicity in construction, practicality, easy maintenance, stability, and convenience. However, these systems are also known for their inaccuracy and unreliability. On the other hand, a closed loop control system is a system in which the output affects the input. For an open loop control system to be converted into a closed loop control system, feedback must be included. Closed loop control systems are known to have higher accuracy, larger bandwidth, and less affected by noise compared to open loop control systems. These systems have also disadvantaged such as a greater cost, more complex design, and the requirement of higher maintenance.

The use of feedback in a control system has both advantages and disadvantages. Control of the transient response, minimal steady state error, sensitivity to modeling errors, and disturbance input rejection are some of the advantages. On the other hand, cost, loss of gain, and stability are the disadvantages of using feedback in a system. [3]

\section{LITERATURE REVIEW}

Analysis, Design, and Optimization of Embedded Control Systems (2016), a study conducted and authored by Amir Aminifar, sheds some light the embedded control or cyber-physical systems wherein a lot of the control applications have the same processing unit. The thesis focuses on the co-design problem of control-scheduling, where the parameters of the controller and scheduling are optimized. New methodologies for the optimization process of embedded control systems are needed to reduce the problems caused by the differences between control applications and traditional embedded applications. Furthermore, the said study covers not only the offline but also the online methodologies for embedded control systems. With that, a control-scheduling co-design methodology is proposed in the study to modify and improve control performance with the maintenance of stability. [4] 
Systems with Limited Feedback Information (2005) conducted by Qiang Ling pays attention to the operation of control systems. However, this thesis concentrates on the problem of having limited feedback information. According to the study, there are two types of feedback limitation dropout and quantization. Dropout implies the missing of the desired feedback measurement, while quantization demonstrates that the desired feedback measurement is reported by a finite number of bits. This study provides an analysis of the effects of both dropout and quantization limitations on the performance and stability of control systems. Moreover, the study suggests development methods that optimize system performance despite feedback information limitations [5].

The study Output Feedback Event-triggered Control (2014) by Mahmoud Abdelrahim focuses on the development of an emulation-based design made for output feedback event-triggered controllers to maintain a set of nonlinear systems. Furthermore, it presents a co-designing methodology to design the output feedback law and the event-triggering condition simultaneously for the lessening and reduction of transmissions. Stabilization of event-triggered controllers for nonlinear systems is also one of the primary objectives of this thesis study. The presentation of this study is coherent and concise as it provided a brief summary of all the concepts and approaches related to the topic. It also discusses the emulation design for nonlinear systems, co-design for LTI systems, and singularly perturbed systems. Despite the major contributions of the research, possible extensions of the study can be an examination of the robustness of the triggering mechanism proposed or an investigation on singularly perturbed systems wherein stability is unnecessary for the dynamics. Most importantly, the research study has investigated the synthesis of the stabilization of output feedback event-triggered controllers linear and nonlinear systems [6].

System Identification for Feedforward and Feedback Control (2016) by Frank Boeren presents separate contributions concerning both feedback control and feedforward control. For feedback control, there are two entries of contributions both for the purpose of developing the specification of the optimization criterion for the combination of model-based feedback controllers. The first one is a proposal of identification for control approach which is applicable for positioning systems characterized by flexible dynamic behavior. This identification proposal for control procedure is made to cover a class of systems to reduce conservatism in the guarantee of performance and stability. The second contribution is a newly designed approach of non-diagonal weighting functions for positioning systems with flexible dynamic behavior. Conventional weighting function designs utilize diagonal weighting functions, based on the inference that the system behaves as a rigid body. The achieved development of the performance is demonstrated through an experiment of a motion system. On the other hand, a novel modeling approach is proposed for the identification of feedforward control. This identification can obtain high servo performance and flexibility with respect to changing tasks. Experimental results of this study verify the advantages of the proposals compared to existing methods for different systems operating in semiconductor manufacturing. [7]

\section{THEORETICAL CONSIDERATION}

There are different needs for feedback and control systems, a good example is for the use of counteracting the disturbance signals which are affecting the output that is desired, another is for innovating as well as improving the performance of a given system which is present in the model uncertainty, a good example of this is for open-loop control which is usually used for processes or machines, the idea behind this is that it specifies the desired reference output signal, aside from this it also computes the input signal which is needed.

Some other theories to be noted for feedback and control systems are the technology of actuators, sensors, and controllers. The application of actuators, sensors, and controllers may become a limitation to the control theory [8].

Actuators may be defined as devices which are capable of manipulating the control variables that are present in a given process. A good example of this is a home temperature control system, the actuators in this system are the electric baseboard heaters and heat pumps. For process controls, however, the usual actuators used are valves, electric motors, and heaters [8].

Sensors, on the other hand, may be defined as the devices which are able to measure the output variables that may be controlled. The most common sensors used are thermocouples which are used for measurements regarding temperature, differential pressure transmitters, radar range sensors, and magnetic flowmeters [8].

Lastly, controllers may be defined as devices which use the feedback measurements from the output variables in order to compute as well as to give control signals to the actuators. In the early days, controllers were mechanical devices, but today microprocessors are now used. Most controllers in our current generation are now implemented with the use of industrial computers or Programmable Logic Controllers or PLC's [8].

Feedback plays an important role in the performance of control systems, although for the case of electric motors, the 
control continues to be of the question since the usual conventions that are related with the control of motor are most of the time constrained with considerations relating to cost, thus the feedback component is eliminated. These Systems can be further optimized by using the Rough Set Theory $[9,10]$.

For the case of electric motors, the AC motor of Nicola Tesla was well understood, and the design did not use much feedback. It is also good that motor speed is derived from the frequency of the power that is supplied subtracted from the losses that are dependent on the given information of the construction of the rotor as well as the effect on it by the specific load. Standard AC motors also have little amounts of slip in the rotor that ranges in between $1800 \mathrm{rpm}$ and 1750 rpm and this shows that the losses in the magnetizing current for the motor as well as the magnetic features in the rotor that are needed to be maintained in perfect synchronization together with the line frequency.

If this is looked with the standpoint of control systems, feedback is considered last and are the slowest loop present in how it is controlled in the motor. In the case of position control, it can be seen that it makes sense since it is normally executed in a PLC or with the use of a motion controller, but at the same time it also makes load regulation challenging since the error detection of the control system is done with a level removed from the load that is present in the system.

Another thing to note is the input and output relationships in a feedback control system. From Feedback Control Theory, the input-output relationship is discussed with the use of a linear system with input $\mathrm{u}$, output $\mathrm{y}$, and transfer function $\mathrm{G}$. Shown below are two tables that relate input and output to one another. [11].

Table 1: Output norms and power for two points

\begin{tabular}{|l|cc|}
\hline & $u(t)=\delta(t)$ & $u(t)=\sin (\omega t)$ \\
\hline$\|y\|_{2}$ & $\|\hat{G}\|_{2}$ & $\infty$ \\
$\|y\|_{\infty}$ & $\|G\|_{\infty}$ & $|\hat{G}(j \omega)|$ \\
$p o w(y)$ & 0 & $\frac{1}{\sqrt{2}}|\hat{G}(j \omega)|$ \\
\hline
\end{tabular}

Table 2: System Gains

\begin{tabular}{|l|ccc|}
\hline & $\|u\|_{2}$ & $\|u\|_{\infty}$ & $\operatorname{pow}(u)$ \\
\hline$\|y\|_{2}$ & $\|\hat{G}\|_{\infty}$ & $\infty$ & $\infty$ \\
$\|y\|_{\infty}$ & $\|\hat{G}\|_{2}$ & $\|G\|_{1}$ & $\infty$ \\
$\operatorname{pow}(y)$ & 0 & $\leq\|\hat{G}\|_{\infty}$ & $\|\hat{G}\|_{\infty}$ \\
\hline
\end{tabular}

\section{DESIGN CONSIDERATION}

Different processes can be represented through block diagrams. For example,

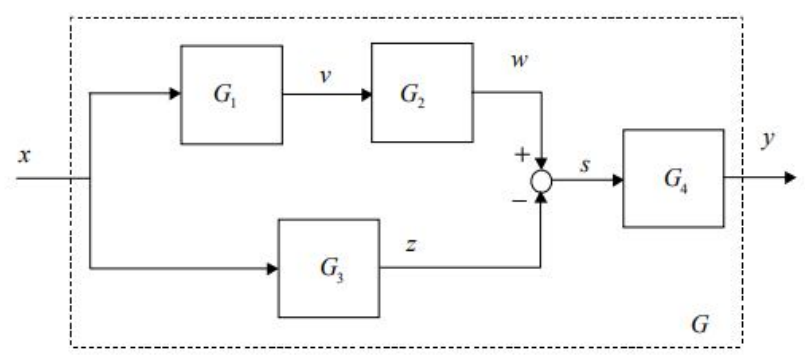

Figure 1: System Block Diagram

The diagram above shows the operation of the system with $\mathrm{x}$ as the input variable and $y$ as the output. The output of the system can be controlled by varying the input. There are three kinds of interconnection in system block diagrams. First is the cascade interconnection which is shown by the relation of blocks G1 and G2. Next is the parallel interconnection which is shown by the connection of G3 with blocks G1 and G2. Lastly, the feedback interconnection sends back a signal to the input to control the output of the system. The values of the system when placed in a database will have large amounts of data. Its database configuration will be like $[12,13]$. The G1and G2 is shown in the figure below.

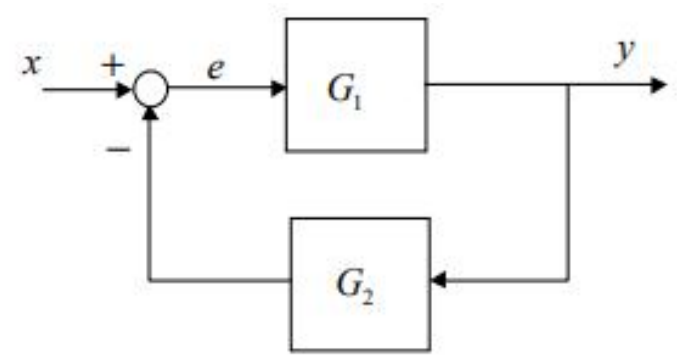

Figure 2: Feedback Interconnection Block Diagram 
Control systems are used in everyday devices. Cruise control with spatial systems in-car systems is responsible for maintaining the desired speed while in highways [14]. This helps in ensuring that the driver is following the speed limit and improving the safety of those on board. The block diagram below shows the process involved in maintaining cruising speed. The input is the desired speed of the driver and the output is the actual speed of the car. The feedback interconnection is responsible for informing the controller if the car is still maintaining its cruising speed [15]. If the controller detects that the speed of the car goes over the desired speed, it will slow down the car back to the desired speed. On the other hand, if the car falls below the desired speed, the controller will command the car to speed up to meet the speed that is required.

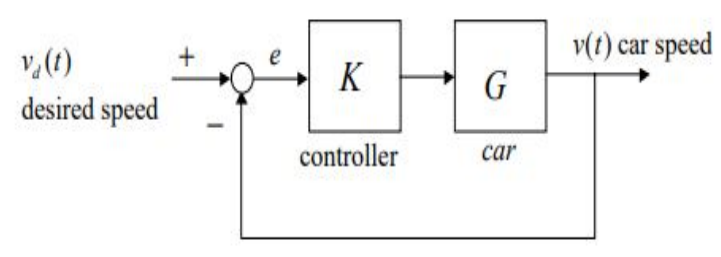

Figure 3: Cruise Control Block Diagram

\section{CONCLUSION}

Nowadays, control systems play a major role in our society and daily living as part of the advancement of technology. Almost every system that surrounds us and helps us every day is a control system. Thus, the research studies and practical applications of control systems must be continued in order to cope up with the emerging of modern technology. As this study discussed, the use of feedback in control systems is essential but has advantages and disadvantages. As a researcher, one must know and understand these factors first before fully implementing control systems with feedback.

Utilizing the system block diagrams as a reference to further simplify the process is also essential to improve the current system that is being implemented in different devices. The block diagram makes the process easier to understand since the stages are straightforward. Keeping in mind some of the theories that are essential in learning and applying control systems are important since actual implementation would continuously fail if theoretical considerations are not included and studied first. It is important to first think and use the given knowledge about the theories before even doing actual work and implementation. The group concluded that in order to better understand and be able to fully implement and make use of the knowledge about a control system in the work field of Electronics and Communications Engineering, both theoretical and design theories as well as knowledge about existing works and the basics of control theory should be well understood first.

\section{REFERENCES}

[1] S. Wu, X. Sun, X. Li, and H. Wang, "On controllability and observability of impulsive control systems with delayed impulses," Mathematics and Computers in Simulation. 2019. https://doi.org/10.1016/j.matcom.2019.03.013

[2] P. E. D. Love, J. Zhou, and J. Matthews, "Project controls for electrical, instrumentation and control systems: Enabling role of digital system information modelling," Automation in Construction. Vol. 103, pp. 202-212, 2019. https://doi.org/10.1016/j.autcon.2019.03.010

[3] Y. Lan, B. Wu, Y. Shi, and Y. Luo, "Iterative learning-based consensus control for distributed parameter multi-agent systems with time-delay," Neurocomputing. Vol 357, pp. 77-85, 2019. https://doi.org/10.1016/j.neucom.2019.04.064

[4] A. Aminafar. "Analysis, Design, and Optimization of Embedded Control Systems." Ph.D. dissertation. Department of Computer and Information Science Linkoping University. 2016. https://doi.org/10.3384/diss.diva-124319

[5] Q. Ling. "Stability and Performance of Control Systems with Limited Feedback Information." Ph.D. dissertation University of Notre Dame. 2005.

[6] M. Abdelrahim. "Output Feedback event-triggered control." Universite de Lorraine. Ph.D. dissertation. 2014.

[7] F. Boeren. "System Identification for Feedforward and Feedback Control with Application in Semiconductor Manufacturing." Philips Innovation Series. 2016.

[8] Y. Li, C. Chen, S. Zhu, and X. Guan, "Sensor scheduling for relay-assisted wireless control systems with limited power resources," ISA Transactions. Vol. 88, pp. 246-257, 2019. https://doi.org/10.1016/j.isatra.2018.11.043

[9] A. Africa and M. Cabatuan, "A Rough Set Based Data Model for Breast Cancer Mammographic Mass Diagnostics." International Journal of Biomedical Engineering and Technology. Vol. 18, No. 4, pp. 359-369, 2015. https://doi.org/10.1504/IJBET.2015.071010

[10] A. Africa, "A Rough Set-Based Expert System for diagnosing information system communication networks." International Journal of Information and Communication Technology. Vol. 11, No. 4, pp. 496-512, 2017. https://doi.org/10.1504/IJICT.2017.10008315

[11] M. Carriegos, N. DeCastro-Garcia, and A. Castaneda, "Partitions, diophantine equations, and control systems." Discrete Applied Mathematics. Vol. 263, No. 1, pp. 96-104, 2019. https://doi.org/10.1016/j.dam.2018.01.015 
[12] D. Ahamad, M. Akhtar, and S. Hameed, "A Review and Analysis of Big Data and MapReduce." International Journal of Advanced Trends in Computer Science and Engineering (IJATCSE). Vol. 8, No. 1, pp. 1-3, 2019. https://doi.org/10.30534/ijatcse/2019/01812019

[13] S. Rao, M. Devi, and P. Kumar, "Wireless sensor Network based Industrial Automation using Internet of Things (IoT)." International Journal of Advanced Trends in Computer Science and Engineering (IJATCSE). Vol. 7, No. 6, pp. 82-86, 2018. https://doi.org/10.30534/ijatcse/2018/01762018

[14] P. Loresco and A. Africa, "ECG Print-out Features Extraction Using Spatial-Oriented Image Processing Techniques." Journal of Telecommunication, Electronic and Computer Engineering. Vol. 10, Nos. 1-5, pp. 15-20, 2018.

https://doi.org/10.1016/j.eswa.2019.01.043

[15] W. Mendes, F. Araújo, R. Dutta, and D. Heeren, "Fuzzy control system for variable rate irrigation using remote sensing." Expert Systems with Applications. Vol. 124, pp. 13-24, 2019. 